\title{
Historia breve de cuatro asentamientos rarámuri \\ de la ciudad de Chihuahua
}

\section{Brief history of four Raramuri settlements in the city of Chihuahua}

\author{
Nelson Solorio Talavera* \\ Jesús Adolfo Trujillo Holguín***
}

* Estudiante en el Doctorado en Educación, Artes y Humanidades de la Universidad Autónoma de Chihuahua. Es licenciado en Letras Españolas por la Universidad Autónoma de Chihuahua y Maestro en Antropología Social por la Escuela de Antropología e Historia del Norte de México. En su trabajo de investigación de la maestría abordó el tema "Los espacios otros. Los rarámuri y los chabochi en la ciudad de chihuahua" y actualmente su investigación gira en torno a la participación de los rarámuri en la educación escolarizada. Correo electrónico nelson.sol.tal@gmail.com

(D) https://orcid.org/0000-0003-1187-2229

** Profesor-Investigador de la Universidad Autónoma de Chihuahua (México). Es doctor en educación por la Universidad Autónoma de Chihuahua y Maestro en Educación por la Universidad Pedagógica Nacional. Entre sus publicaciones recientes están: La educación en Ranchería Juárez, Chihuahua, $1932-2018$ (2019) y Debate legislativo y educación. El artículo tercero a cien años de la Constitución Política de 1917 (coord.) (2018). Cuenta con reconocimiento al perfil Prodep y del Sistema Nacional de Investigadores Nivel 1. Es socio del Consejo Mexicano de Investigación Educativa, de la Red de Investigadores Educativos Chihuahua y presidente de la Sociedad Mexicana de Historia de la Educación. Entre sus temas de interés están la historia e historiografía de la educación y formación de maestros. Correo electrónico: jatrujillo@uach.mx

\section{https://orcid.org/0000-0002-6738-1878}

Historial editorial

Recibido: 24-julio-2019

Aceptado: 05-noviembre-2019

Publicado: 31-enero-2020

ISSN-e: 2594-2956 
Historia breve de cuatro asentamientos rarámuri de la ciudad de Chihuahua

\section{Resumen}

El siguiente texto es resultado del trabajo de campo etnográfico realizado en 2017, destinado a conocer las condiciones en que habitan los rarámuri de la ciudad de Chihuahua. En este artículo se recogen, además de observaciones etnográficas, comentarios y testimonios de diversos actores rarámuri y no-indígenas (chabochi), en torno a la formación y desarrollo de cuatro asentamientos en la ciudad de Chihuahua, que en la actualidad cuentan con alrededor de 800 habitantes. Su objetivo es ilustrar sobre el devenir y las condiciones de vida que enfrentan los residentes de esta etnia en el entorno urbano. Así mismo se esbozan algunos tópicos y problemáticas que este grupo indígena enfrenta cotidianamente, tales como son la falta de recursos económicos, adicciones, violencia y constantes intervenciones de los no-indígenas en sus espacios físicos y sus decisiones como grupo, que determinan la educación y la formación de su propia subjetividad.

Palabras clave: Asentamientos, rarámuri urbanos, historia oral, intervención institucional, formación de subjetividades. 


\title{
Brief history of four Raramuri settlements in the city of Chihuahua
}

\begin{abstract}
The following text is the result of the ethnographic field work carried out in 20I7. Its purpose was to know the conditions in which groups of Raramuri live in the city of Chihuahua. This article includes ethnographic observations, comments and testimonies of various actors, rarámuri and non-indigenous (chabochi) people with respect to the formation and development of four rarámuri settlements in Chihuahua city of around 800 inhabitants each. The objective is to illustrate the life style and living conditions faced by Raramuri residents of this urban environment. Moreover, in this text there's an outline of some topics and problems faced daily by this ethnic group, such as the lack of economic resources, addictions, violence, as well as the constant non-indigenous intervention in their physical spaces. It also shows the decisions taken as a group that determine the education and formation of the subjectivities of these urban raramuri.
\end{abstract}

Keywords: Settlements, urban rarámuri, oral history, institutional intervention, formation of subjectivities. 
Bref historique de quatre colonies de Raramuri dans la ville de Chihuahua

\section{Résumé}

Le texte suivant est le résultat du travail ethnographique de terrain réalisé en 20I7, dont le but était de connaître les conditions dans lesquelles les Raramuri habitent la ville de Chihuahua. Cet article comprend, en plus des observations ethnographiques, des commentaires et des témoignages de divers acteurs, rarámuri et non indigènes (chabochi), sur la formation et le développement de quatre colonies rarámuri dans la ville de Chihuahua, qui ont actuellement Environ 800 habitants. Son objectif est d'illustrer l'avenir et les conditions de vie des habitants de Raramuri dans cet environnement urbain. Dans ce texte sont également décrits certains sujets et problèmes auxquels ce groupe ethnique est confronté quotidiennement, tels que le manque de ressources économiques, les dépendances, la violence, ainsi que l'intervention non indigène constante dans leurs espaces physiques et leurs décisions en tant que groupe, qui ils déterminent l'éducation et la formation des subjectivités du rarámuri urbain.

Mots-clés: Colonies, Formation des subjectivités, Historie orale, Intervention institutionnel, Rarámuri urbain. 
Krótka historia czterech osiedli Rarámuri w mieście Chihuahua

\section{Streszczenie}

Poniższy tekst jest wynikiem etnograficznych prac terenowych przeprowadzonych w 2017 r., których celem było poznanie warunków, w jakich indianie Rarámuri zamieszkują miasto Chihuahua. Artykuł ten zawiera, oprócz obserwacji etnograficznych, komentarzy i świadectw różnych podmiotów, rarámuri i bialych (chabochi), wokół powstawania i rozwoju czterech osiedli Rarámuri miasta Chihuahua, które dziś mają około 800 mieszkańców. Jego celem jest zilustrowanie warunków życia mieszkańców Rarámuri w środowisku miejskim. W tekście przedstawiono rowniez problemy, z którymi ta grupa etniczna boryka się na co dzień, takie jak brak zasobów ekonomicznych, uzależnienia, przemoc, a także stała interwencja bialych na decyzje grupowe takie jak edukacja i kształtowanie tozsamosci miejskich Rarámuri.

Slowa kluczowe: Osady, Rarámuri, historia mowiona, interwencja instytucjonalna, tworzenie podmiotów 


\section{Antecedentes}

La residencia de los rarámuri en la ciudad ha sido abordada principalmente desde la antropología y contamos con los notables trabajos de Morales (2013), quien aborda el tema de la intervención de las instituciones no-indígenas -externas al asentamiento- y la manera en que estos espacios son etiquetados como una célula urbana en la cual es necesario promover el progreso. Posteriormente, Morales (2014), en un trabajo más amplio hace un recorrido por los diferentes aspectos de la vida en los asentamientos, en el que se entrelazan el género y la etnicidad como herramientas para el análisis.

Además de los trabajos antes mencionados, resultan importantes otros investigadores que ayudan a conocer mejor a los rarámuri, como residentes de la ciudad. Para la antropóloga Fernández (2013), los asentamientos son núcleos residenciales centrales en los procesos migratorios y en el establecimiento de redes sociales rarámuri. Mientras que Herrera (2013) desarrolla una discusión sobre el concepto de comunidad e identidad y en su obra señala la idea de que los asentamientos están fundados en conceptos e interpretaciones noindígenas sobre lo que es o tiene que ser una "comunidad". A su vez los no-indígenas que intervienen en el asentamiento "adocenan la vida comunitaria tarahumara, reduciéndola a fórmulas huecas de escaso mérito" (Herrera, 2013, p. 192). Estos espacios se nos presentan como comunidades rarámuri impostadas o forzadas, insertas en la ciudad.

Podemos observar que las tres posturas de estos autores indagan sobre la relación que existe entre estos espacios rarámuri y el entorno urbano, apuntando hacía la idea de que los espacios habitados por esta etnia en

116 la ciudad de Chihuahua son lugares definidos por su interacción con sujetos e instituciones no-indígenas.

\section{Los “asentamientos tarahuamaras"}


En la ciudad de Chihuahua el término asentamiento rarámuri, indígena o tarahumara es usado para denominar a cualquier espacio en el que resida una concentración de población perteneciente a las etnias procedentes de la Sierra Tarahumara, de las cuales la mayoría es población rarámuri. Los censos realizados por la Coordinación Estatal de la Tarahumara -actual Comisión Estatal de los Pueblos Indígenasemplean ese término para referirse a estos espacios, aunque sólo tengan cinco residentes (Fernández, 2013). Entonces, existen vecindades, barrios y pequeños núcleos donde viven sólo familias rarámuri y estos lugares suelen ser identificados desde el punto de vista de las instituciones públicas y privadas como asentamientos indígenas. Además, el término ha sido asimilado en el lenguaje coloquial, de manera que los vecinos y los mismos residentes suelen señalar a las vecindades y los barrios rarámuri como asentamientos.

Generalmente los residentes de estos espacios se han trasladado temporal o permanentemente al entorno urbano, por lo que se les suele considerar poblaciones migrantes. Este traslado o migración lo realizan valiéndose de redes familiares o de paisanazgo (Fernández, 2013).

Así pues, la palabra asentamiento refiere a un emplazamiento habitado por una población que se ha trasladado desde un territorio original, por lo que su empleo tiene un peso político y etiqueta a los rarámuri como migrantes. Al hablar de asentamientos no se toman en cuenta las condiciones concretas de cada espacio, ni si sus residentes son población migrante o si son personas que tienen 30 años o toda su vida en la ciudad de Chihuahua. Sin embargo, es el término usual, un concepto nativo, característico del léxico en ciertos ámbitos de la ciudad de Chihuahua, bajo el cual son etiquetados estos emplazamientos. La utilidad del término asentamiento consiste en que nos permite señalar la particularidad de un fenómeno cultural, una manera de dotar al espacio de significado proveniente de la interacción entre grupos rarámuri y no-indígenas. 
Entonces, los asentamientos tarahumaras en la ciudad de Chihuahua son espacios definidos bajo un criterio étnico. En este sentido, son la expresión urbanística del encuentro y la convivencia, así como de la marginación y la segregación que operan en el proceso de las relaciones entre los rarámuri y los no-indígenas. Todos fueron establecidos en los márgenes de la ciudad, o en colonias tradicionalmente habitadas por pobladores de bajos recursos económicos.

Tabla: Asentamientos rarámuri de la ciudad de Chihuahua.

\begin{tabular}{|c|c|c|c|}
\hline \multicolumn{3}{|c|}{ Año de fundación } & $\begin{array}{l}\text { Población } \\
\text { aproximada en } \\
\text { el año } 2020\end{array}$ \\
\hline $\begin{array}{l}\text { El Oasis / Garí } \\
\text { Rosácame }\end{array}$ & $\begin{array}{l}\text { I957 (Probablemente } \\
\text { los rarámuri se } \\
\text { asentaron en ese } \\
\text { espacio antes de la } \\
\text { intervención de los } \\
\text { religiosos). }\end{array}$ & $\begin{array}{l}\text { Sacerdote Jesuita o } \\
\text { ministro protestante. A } \\
\text { este respecto son } \\
\text { ambiguos los datos. }\end{array}$ & 520 \\
\hline Pino Alto & $\begin{array}{l}\text { I974 (Aunque hay } \\
\text { testimonios que } \\
\text { consideran que es de } \\
\text { una fecha cercana a } \\
\text { I960). }\end{array}$ & $\begin{array}{l}\text { Iniciativa de una } \\
\text { mujer laica católica } \\
\text { que daba albergue a } \\
\text { familias procedentes } \\
\text { de la Sierra. } \\
\text { Posteriormente, el } \\
\text { asentamiento pasó a } \\
\text { manos de la Compañía } \\
\text { de Jesús. }\end{array}$ & $\mathrm{I} 2 \mathrm{O}$ \\
\hline $\begin{array}{l}\text { Colonia } \\
\text { Tarahumara }\end{array}$ & I99I & $\begin{array}{l}\text { El gobierno municipal } \\
\text { (presidente Patricio } \\
\text { Martínez) desplazó a } \\
\text { varias familias que se } \\
\text { habían asentado cerca } \\
\text { del centro de la } \\
\text { ciudad, hacia una zona } \\
\text { en aquel entonces } \\
\text { despoblada. }\end{array}$ & 380 \\
\hline $\begin{array}{l}\text { Siyóname } \\
\text { Churuwi / } \\
\text { Pájaro Azul o } \\
\text { Sierra Azul }\end{array}$ & I992 & Iniciativa jesuita. & 300 \\
\hline $\begin{array}{l}\text { Carlos Diaz } \\
\text { Infante }\end{array}$ & $\begin{array}{l}2006 \text { (Es el año en que } \\
\text { se estableció } \\
\text { formalmente el }\end{array}$ & Iniciativa jesuita. & I5O \\
\hline
\end{tabular}

Debates por la Historia - Artículos 


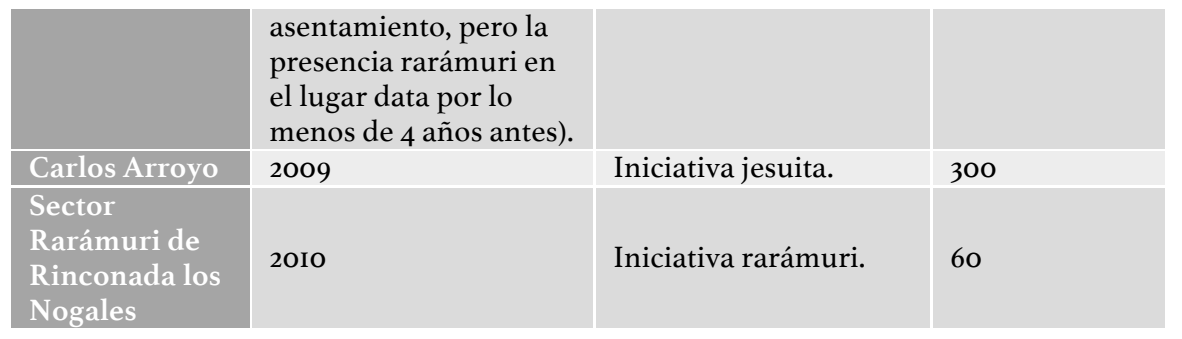

Fuente: Basada en datos de Morales (2013, p. 26), pero se complementa con números que reflejan el crecimiento demográfico de los asentamientos, así como algunos comentarios sobre los años y la iniciativa para la fundación.

Nota: El número de los pobladores es impreciso debido a que hay una fracción de población flotante considerable en todos los asentamientos.

Como se aprecia en la tabla I, los asentamientos fueron fundados (y son administrados) por iniciativa de autoridades religiosas, gubernamentales o particulares no-indígenas. Se puede decir que la intervención institucional de los no-indígenas en los espacios de residencia rarámuri no cesa con la fundación, sino que persiste en toda clase de medidas administrativas. Esta intervención de instituciones noindígena o chabochi $i^{I}$ al interior de los asentamientos es notable y cotidiana. De acuerdo con Morales (2013) las intervenciones institucionales en el asentamiento rarámuri El Oasis etiquetan y tienen una carga simbólica que los concibe como sujetos con necesidades constantes y como lugar cuyas condiciones justifican la caridad y las acciones gubernamentales. El autor señala que la intervención institucional presentarse al asentamiento como un espacio que no participa del "progreso" y representa una de las maneras en que la residencia rarámuri en la ciudad funciona como un factor de diferenciación entre ambos grupos. Pero (en contraste con la vestimenta y el idioma) el emplazamiento de asentamientos especializados para acoger a la población rarámuri se lleva a cabo con la intensa y explícita participación de instituciones chabochi. 
Esta intervención o participación de las instituciones al interior de El Oasis tiene el efecto de etiquetar y diferenciar a este espacio del resto de la ciudad. Lo constituye como una célula en la que se ha concentrado al menos parte de la población rarámuri. Entonces, cómo muchos de los elementos que caracterizan a El Oasis, no proceden de la iniciativa rarámuri, sino de actores e instituciones chabochi, podemos hablar de que además de ser un factor de diferenciación, el asentamiento es construcción de la otredad, en el sentido de que el grupo chabochi, valiéndose de mayor acceso a los recursos del poder (instituciones públicas y privadas) controla al menos parte de las condiciones bajo las cuales los rarámuri residen en la ciudad. Estos enunciados sobre El Oasis -el asentamiento indígena más antiguo de la ciudad- son generalizables al resto de los espacios que, al igual que este, fueron fundados por la iniciativa no-indígena para acoger a los rarámuri que habitan la ciudad.

\section{Una historia oral}

A pesar de esta intervención institucional, desde la fundación hasta el desarrollo y la vida cotidiana de los asentamientos, la historia carece de un registro oficial y se encuentra dispersa en la memoria de sus participantes, algunos investigadores y unos pocos documentos. Esta carencia de registros parece estar relacionada con la condición de marginación e incluso clandestinidad en la que surgieron algunos de los asentamientos rarámuri de la ciudad de Chihuahua.

A pesar de no pertenecer a la historia oficial de las instituciones del Estado o la Iglesia, los asentamientos han sido muy significativos para la formación y la historia de vida de los rarámuri urbanos por más de 60 años. Su abordaje requiere de los métodos de la historia oral, con el fin de conocer cómo es que personas concretas se enfrentan a la cotidianeidad y al devenir de la vida en estos espacios (Moss, I99I).

En el caso particular de esta investigación, la recopilación de datos etnográficos como el número de habitantes y la etnicidad de los 
habitantes de los asentamientos, se realizó mediante entrevistas con distintos actores del proceso de fundación y desarrollo de estos espacios. Las personas que aquí son citadas dieron el consentimiento para la aparición de su nombre y los materiales mencionados más adelante fueron publicados por la Asociación Asentamientos Tarahumares. A partir de estas fuentes se reconstruyó un relato sobre la fundación de cuatro de los más grandes núcleos habitacionales rarámuri de la ciudad de Chihuahua.

Así, en los siguientes apartados encontraremos fuentes testimoniales y referencias a documentos que dialogan entre sí y nos permiten el empleo de la memoria de los sujetos como fuente para la historia reciente de los rarámuri urbanos (Lara, 20I0). Este hilar de las distintas voces se realiza a través de la narración en primera persona por parte de uno de los autores de este artículo, siendo elegida esta perspectiva porque se busca ilustrar la manera en que la interacción humana, la presencia del investigador, genera el discurso desde su propia subjetividad.

\section{Historia de cuatro asentamientos rarámuri}

\section{Primeros asentamientos}

Alrededor de 1990, tras concluir su servicio en la Misión de la Tarahumara, el sacerdote jesuita Carlos Díaz Infante fue transferido a la ciudad de Chihuahua. Para entonces -como ahora- los ideales de la Compañía de Jesús incluían la proximidad con la cultura y el idioma de los pueblos en los que cumplían misión evangélica (Mireles, 2012), por lo que después de 40 años en las montañas, el Padre Díaz Infante era un hablante fluido del idioma rarámuri. Además -como era de esperarsesentía apego hacia la gente que había sido su preocupación durante décadas y esto se hizo evidente cuando pidió a las autoridades eclesiásticas que le concediera continuar su labor misional -esta vezcon los rarámuri de la ciudad. 
Lo más cercano a un modelo en el que habrían de vivir los tarahumaras migrantes era El Oasis, un espacio controlado y cercado al que se le llegó a poner el letrero de "asentamiento tarahumara católico" (Morales, 2013, p. 24). Así, siguiendo una configuración similar, en la que la posición de la capilla tenía un lugar preponderante, se construyó el asentamiento Siyóname Churuwí en I992, también llamado Sierra Azul o el Pájaro Azul.

En el extremo suroriental de la ciudad de Chihuahua, en un terreno ejidal prestado, fue a vivir Carlos Díaz Infante con un grupo rarámuri que había sido desplazado del centro de la ciudad, ya que se encontraban en "terrenos irregulares" (F. Ruiz, comunicación personal, I8 de abril, 20I7). ${ }^{2}$ Las personas fueron desplazadas de áreas próximas a la antigua Central Camionera hacia la colonia Sierra Azul y Díaz Infante fue nombrado párroco de la recién establecida Parroquia de San José de los Tarahumaras en 1993, misma que se compone de al menos 75 comunidades indígenas asentadas en la ciudad de Chihuahua. Esa época fue importante para la historia de los rarámuri que residen en la ciudad de Chihuahua porque también ocurrieron los desplazamientos que dieron pie a la fundación de la Colonia Tarahumara, por iniciativa gubernamental.

Entre 1996 y 1997, Carlos Díaz Infante conoció "a un grupo de familias que estaban en un asentamiento irregular cerca de la Plaza de Toros" (F. Ruiz, comunicación personal, I8 de abril, 20I7), es decir en el área antes mencionada, pero esta vez con motivo de la construcción del parque El Palomar, de donde fueron retiradas familias indígenas y de escasos recursos económicos por iniciativa de Gobierno del Estado. Ante esto, Díaz Infante ayudó para que pudieran establecerse en terrenos de la colonia $\mathbf{I}^{\mathbf{0}}$ de Mayo, los cuales negoció con el ejido Robinson, que ya anteriormente había cedido los terrenos del Siyóname Churuwí. Así, "con láminas de cartón, con láminas de zinc, con pedazos de madera, etc., construyen sus viviendas de manera muy precaria" (F. Ruiz, comunicación personal, I8 de abril, 20I7). Según otras fuentes, los primeros en establecerse en el terreno de la $I^{\circ}$ de Mayo 
fueron una familia procedente de Carichí, de donde llegan muchos residentes del asentamiento (Fernández, 2013). Posteriormente, en 2003, el padre Díaz Infante falleció.

\section{Asentamientos Tarahumares AC}

La Compañía de Jesús encargó a Carlos Arroyo Herrera ${ }^{3}$, otro sacerdote veterano con décadas de trabajo en la Sierra Tarahumara, continuar la búsqueda de financiación para mejorar las condiciones de vida en el nuevo asentamiento de la colonia $\mathrm{I}^{\mathrm{O}}$ de Mayo, que fue nombrado Asentamiento Tarahumar Carlos Díaz Infante S.J. (F. Ruiz, comunicación personal, I8 de abril, 2017).

El sacerdote Carlos Arroyo consiguió fondos para construir cuatro viviendas "las más grandes [de las que constituyen el asentamiento en la actualidad], de dos recámaras, ecológicas, construidas con botes de leche. Pero el hacinamiento y las malas condiciones de vivienda continúan" (F. Ruiz, comunicación personal, I8 de abril, 2017). Entonces, el padre Oscar Reinal, también jesuita que había estado en la Misión de la Tarahumara, comenzó a ayudar en esta labor y logró un enlace -en el año 2006- con el gobernador José Reyes Baeza Terrazas, la presidencia municipal y diversos miembros del empresariado chihuahuense, incluido el ingeniero Francisco Vicke Andrews, quien trabajaba en Cementos de Chihuahua.

La unión de estos tres sectores (Iglesia, gobierno y empresariado) llevó a la fundación de la asociación civil Asentamientos Tarahumares, misma que proporcionó los recursos para terminar el asentamiento Díaz Infante como se encuentra en la actualidad: "con casas demasiado pequeñas, con un material que no es cemento [...]" (F. Ruiz, comunicación personal, I8 de abril, 20I7), material de una calidad inferior que se hincha y pierde su forma con la humedad. Para albergar a nuevas familias, se construyeron más casas de las que necesitaban en ese momento -en total 32- equipadas con baño, camas, un calefactor de gas, refrigerador, estufa, mesa y sillas, fregadero, alacenas, un 
ventilador y un tanque de gas. En el espacio común se construyeron una cancha de básquet, juegos para los niños y se instaló adoquinado. Poco después, en una fosa destinada para sembrar un árbol los residentes improvisaron un fogón que les permite cocinar cuando se lleva a cabo una celebración como las Semana Santa, o más frecuentemente lo emplea alguna familia cuando no tiene gas en la cocina (F. Ruiz, comunicación personal, I8 de abril, 20I7).

Mientras se realizaba la construcción de las viviendas, las familias se retiraron a vivir a otros lugares de manera temporal, pero al regreso cada una pagaba 15 pesos a la semana por el servicio de agua potable y energía eléctrica. En la actualidad esa suma es de 25 pesos, es decir, se trata de un precio accesible aun para personas de bajos recursos económicos.

En ese tiempo también se redactó un reglamento en el que se establecían las condiciones ideales que, según los jesuitas, habrían de fortalecer la cultura rarámuri en la ciudad: Se prohibían "las borracheras", las visitas demasiado largas (para prevenir que se saturara el asentamiento), los niños tenían que ir a la escuela regularmente, las vacunas debían aplicarse a todos los residentes y establecieron regulaciones que buscaban promover una sana alimentación. "Eso es lo que ellos se imaginaron que debía ser" (F. Ruiz, comunicación personal, I8 de abril, 20I7). Así mismo, se estableció un contrato de comodato, es decir, las casas les fueron prestadas a condición de no dañarlas y cumplir las reglas comunes. Felipe Ruiz explica que en aquel entonces fue invitado a trabajar en la asociación: "No son dueños de ellas. Porque teníamos la experiencia de El Oasis, que fueron comodato en un tiempo, después ya se perdió [...] lo

124 vendieron, lo perdieron, lo apostaron en borrachera, en la teswinada [...] lo que sea." (F. Ruiz, comunicación personal, I8 de abril, 20I7).

El relato de María Luisa Chacarito ${ }^{4}$ añade otra perspectiva a la manera en que se estableció el reglamento. Por aquella época ella llegó a formar parte de las autoridades rarámuri en el Díaz Infante, tenía menos de 25 
años y en su primera junta con los gobernadores (al parecer también acudió el siríame ${ }^{5}$ de El Oasis), los jesuitas de la asociación y gente de la Coordinación Estatal de la Tarahumara les planteó la necesidad de desarrollar un reglamento para los asentamientos. Entonces, el padre Arroyo los invitó a su oficina -a ella y algunos miembros de su familiacon el fin de que lo ayudaran en la elaboración de este instrumento legal. Y estas fueron las reglas que según María Luisa conformaban aquel primer reglamento:

Si una persona trata de violar a un miembro de la comunidad, inmediatamente sería expulsado con toda su familia. Si una persona andaba causando problemas con chismes, se le advertía tres veces y luego era expulsado. La persona que bebe, si llega demasiado ebria, la familia podía no dejarlo entrar hasta que estuviera sobria. Todos debían participar en las fiestas tradicionales. Que no se cambiara al idioma rarámuri por el español. La limpieza era responsabilidad de todos. Y que tenían derecho a quedarse a vivir en las casas el tiempo que quisieran. (M.L. Chacarito, comunicación personal, I3 de marzo 20I7).

En varias conversaciones, María Luisa ofreció distintos matices sobre su percepción de los estatutos bajo los cuales viven en los asentamientos de la asociación. Por una parte, ella fue de las promotoras de esta reglamentación y -sin embargo- reconoce la situación de incomodidad y vigilancia en que son sometidos.

Así pues, Felipe Ruiz quedó a cargo del asentamiento. La asociación lo invitó porque había sido miembro de la Compañía de Jesús y tenía experiencia trabajando en la Sierra Tarahumara. Junto con él, dos mujeres chabochi laicas, motivadas por ideales religiosos, empezaron a trabajar como promotoras de la vida de los indígenas: Estela Parra y Reyna Loera. De esta última María Luisa Chacarito cuenta que llegó al asentamiento por curiosidad, ya que se la había dicho que los rarámuri "eran unos salvajes" y quería comprobarlo, pero en su estancia se dio 
cuenta que lo dicho era mentira (M.L. Chacarito, comunicación personal, I3 de marzo 20I7).

El objetivo del recién formado equipo era fortalecer la educación y la salud (ambas desde el modelo chabochi), así como las tradiciones de los rarámuri. En palabras de Felipe Ruiz: "ofrecerles lo mejor de nuestra cultura sin que pierdan la suya, que ese fue el sueño que yo siempre manejé, buscar que sigan siendo indígenas con el pleno goce de sus derechos y, al mismo tiempo, ofrecerles no lo más barato y lo peor que tenemos, sino tratar de ofrecerles los valores, respeto, educación, servicio. Valores en los que creo y que son lo mejor del ser humano mestizo" (F. Ruiz, comunicación personal, I8 de abril, 20I7).

\section{El asentamiento Carlos Arroyo Herrera}

En el año 2009 se construyó el asentamiento Carlos Arroyo Herrera S.J., esta vez nombrado en honor al recién fallecido párroco de San José de los Tarahumara. Hasta la fecha es el proyecto más ambicioso de la Asociación, con una infraestructura mucho más desarrollada (cuenta con 150 metros de largo y 50 de ancho; a diferencia del Díaz Infante de solamente 59 por 48 metros). Se logró gracias a la iniciativa del padre Óscar Reinal, quien era muy cercano al gobierno municipal de Carlos Borruel, así como al director de Planeación y Evaluación del municipio en aquel entonces, Elías Saad.

Felipe Ruiz, como director del Díaz Infante, se opuso a la construcción del nuevo asentamiento pensando en que, junto con los gastos excesivos de la asociación ${ }^{6}$, significaba que se le prestaría menos atención y recursos al Díaz Infante, cuyas necesidades y gastos no estaban cubiertos:

Lo que teníamos que hacer es generar un capital que soporte los gastos del Carlos Díaz Infante, para ofrecer una nueva experiencia más completa, sin la necesidad de andar haciéndote 
garras para conseguir el apoyo económico que tenían Reyna y Estela (F. Ruiz, comunicación personal, I8 de abril, 20I7).

Este episodio representó un cambio en la naturaleza de la Asociación pues ahora el nuevo personal contaba con sueldos y prestaciones, mientras que sus fundadores y el personal que trabaja en el Díaz Infante, seguían haciendo un trabajo voluntario, basado en los ideales y valores de la Compañía de Jesús. La injerencia más profunda del empresariado (vinculado también a la derecha política del Partido Acción Nacional) llevó a estos cambios en que los valores de la compañía de Jesús fueron sustituidos por las prioridades economicistas, que operaban en términos de desarrollo; ante lo cual la asociación debía de expandirse, crecer: "Aquí se privilegió la cantidad en lugar de la cantidad" -dice Felipe Ruíz- quien ve aquel giro como el inicio de su rompimiento con ella: "Yo había imaginado un asentamiento que no fuera carga para los indígenas, que fuera una vivienda digna, que les permitiera realizar su vida, como ellos quieran" (F. Ruiz, comunicación personal, I8 de abril, 20I7).

\section{La participación de la iniciativa privada}

Cuando estuvieron terminadas las obras del Carlos Arroyo, hubo nuevos cambios en la asociación. Arturo Ugalde sustituyó al ingeniero Vicke como presidente. El personal se redujo al mínimo, se dejaron de rentar oficinas en el centro de la ciudad y la dirección de AsenTar A.C. se estableció en el Carlos Arroyo, a unos tres kilómetros del Díaz Infante y otros tantos del Siyóname Churuwí, en el margen suroriental de la mancha urbana de la ciudad de Chihuahua.

A diferencia de los otros dos asentamientos, el Carlos Arroyo no se formó a partir de un grupo de familias conocidas entre sí, o bien unidas bajo una misma necesidad emergente, como fueron los desalojos de los años 90. Desde la perspectiva de Felipe Ruiz esto generó un problema nuevo, la falta de cohesión en la comunidad: "Se tomó de aquí y allá, gente que llenaba ciertos parámetros. Pero no había, como en el Díaz 
Infante, gente más homogénea, que se conocían porque vivían juntos desde antes, y esto da un soporte que no tiene un conglomerado que juntaste de aquí y de allá..." (F. Ruiz, comunicación personal, I8 de abril, 20I7).

De aquella etapa de selección de los residentes queda una especie de encuesta que denominaron "estudio socioecónomico" y que se siguió aplicando de la misma manera, año tras año hasta el 20I6, con el fin de mantener la información actualizada de los residentes. Es decir, había una aparente desconfianza entre la asociación y la gente a la que quería beneficiar (en contraste con la gran proximidad que existía entre el padre Díaz Infante y los rarámuri). Había una distancia enorme y un desconocimiento que no se pretendía salvar mediante la proximidad, sino mediante el control. Esto no quiere decir que los jesuitas no controlaran, sino que lo hacían con otros medios, acaso menos directos que la amenaza jurídica. Este fue un cambio en la manera en que los chabochi se relacionaban con los rarámuri de los asentamientos mediante otro tipo de autoridad donde la formalidad se modificó. El discurso religioso y moral, fue dejado en segundo plano, en nombre del discurso jurídico y económico.

Este cambio no se limitó a la manera de seleccionar los residentes. $\mathrm{Su}$ principal efecto repercutió en los objetivos expresos de los asentamientos y de la asociación. En una entrevista de radio realizada en 20I4, Arturo Ugalde declaró: "La tendencia actual en todo México [...] es buscar la sustentabilidad y en Chihuahua nos estamos adelantando" (Ugalde, 20I4). Es decir, se privilegiaron iniciativas que buscaban el establecimiento de diversos proyectos productivos que operaran de manera localizada en el Carlos Arroyo y el Díaz Infante. 128 Idealmente esto les brindaría independencia económica a los residentes, aunque por otro lado no dejaron de aceptar las donaciones y las iniciativas altruistas, que siguen siendo la principal fuente de recursos para la Asociación. 
Para esas fechas, Eleazar Sánchez Ramos -quien compartía los anteriores objetivos- fue nombrado director de la Asociación. Bajo su administración se construyó un invernadero y se criaban chivas en el asentamiento Carlos Arroyo, con el objetivo de comercializar productos lácteos. Así mismo, se construyó la Escuela Tarahumara de Artes y Oficios Óscar Reinal S.J.; todo bajo la primicia de volver a los asentamientos lugares productivos.

En diciembre de 2015 se difundió un video elaborado por la misma Asociación en el que solicitaban fondos "para ser autosustentable, arrancar en forma y comenzar a generar sus propios recursos”, decía una voz en off, mientras se mostraban imágenes del Carlos Arroyo (Asentamientos Tarumares, 20I5). En ese mismo video, Ugalde solicita la cooperación de la sociedad en general "para poder obtener una redituabilidad en el sostenimiento de la misma y la generación de mejores ciudadanos tarahumaras" (Ugalde en Asentamientos Tarumares, 20I5); mientras que Eleazar Sánchez dice: "Somos acompañantes [...], acompañamos a las familias en este proceso de integración o de asimilación que vienen a presentar aquí en las zonas urbanas" (Sánchez en Asentamientos Tarumares, 20I5). En estas declaraciones no solo se lee el cambio en el enfoque económico de los asentamientos, sino también en el ideal chabochi de lo que un rarámuri debería ser. Ugalde habla en términos de redituabilidad y ciudadanía; y Eleazar Sánchez recurre a los tópicos de la integración y asimilación; que se contraponen a los fundamentos de las misiones jesuitas que enfatizan el respeto por la cultura.

Podemos observar una tensión entre dos concepciones del sentido de los asentamientos rarámuri urbanos, pero no un rompimiento del modelo colonial, ya que las misiones que los jesuitas establecieron en la Nueva Vizcaya tenían un eminente carácter económico y su objetivo era proveer de mano de obra a la minería y la agricultura que mantenía al Imperio Español (Cramaussel, I99I y Deeds, 2003). Ese carácter económico de las misiones disminuyó a lo largo del siglo XX y principios del XXI, hasta el punto que los asentamientos, no sirven a un 
proyecto productivo o económico particular, sino que -como podemos apreciar en los intentos de la Asociación por integrar a los rarámuri al mercado laboral- se encuentran inmersos en las dinámicas comunes del llamado capitalismo tardío o neoliberalismo.

Sin embargo, bajo los ideales de la Compañía de Jesús, los asentamientos eran ante todo lugares de residencia y se esperaba que se sostuvieran mediante la caridad de un patronato cercano a la Iglesia y con el trabajo que realizaba cada rarámuri adulto de manera independiente. Dice Felipe Ruiz:

Yo no pensaba en términos de autosustentabilidad y proyectos productivos, yo decía: Todos en el Díaz Infante tienen trabajo, todas las mujeres solas tienen trabajo. No son los grandes trabajos [...] el trabajo en la obra, en una granja, en el campo piscando como jornaleros, las mujeres en el servicio doméstico, que no tenían prestaciones, en fin [...] Sí tenían situaciones laborales mínimas, pero todos tenían resuelta su situación económica (F. Ruiz, comunicación personal, I8 de abril, 20I7).

El mismo enfoque quiso aplicarse en el Díaz Infante, pero Felipe Ruiz se opuso, argumentando lo expresado en la cita anterior y agregando que los proyectos productivos como los realizaba la Asociación no tenían éxito, principalmente por la dificultad para comercializar los productos. Además, según los testimonios de Ruiz y de Eleazar Sánchez, los proyectos productivos han tenido menos éxito del esperado debido a la poca participación de los rarámuri. Acaso el ejemplo más pertinente sea el de la "Escuela Tarahumara de Artes y Oficios, Óscar Reinal S.J.", la cual a principios de año contaba con solo 7 alumnos entre los residentes del Carlos Arroyo y para complementar un poco la asistencia se trajo gente del Siyóname Churuwí y del Díaz Infante (E. Sánchez, comunicación personal, 3 de febrero, 2017). A la fecha de conclusión del trabajo de campo realizado para esta investigación -abril del 20I7- la escuela dejó de operar por la inasistencia. 
"No quieren", dijo Eleazar con un tono de resignación. Esta aparente indolencia parece difícil de entender, sin embargo, no lo es tanto si tomamos en cuenta el punto de vista de los rarámuri, ellos ofrecen explicaciones de lo más sencillas y convencionales (E. Sánchez, comunicación personal, I3 de febrero, 20I7).

Juan Carlos Plascencia, joven siríame del Carlos Arroyo menciona: “yo quisiera aprender a coser, pero no tengo tiempo", y es que él trabaja en una fábrica de ropa deportiva en el centro de la ciudad donde usan máquinas industriales, pero no tiene tiempo de entrar al taller de costura que ofrecía la Escuela de Artes y Oficios (J.C. Plascencia, comunicación personal, 20 de marzo, 20I7).

Varios jóvenes trabajan o estudian la preparatoria o la universidad. La mayoría de las mujeres se dedican al servicio doméstico, en casas de remotas colonias de clase media y alta; se abastecen de víveres para el consumo diario en mercados populares y supermercados, buscando los mejores precios, aunque para ello tengan que invertir 3 o 4 horas en traslados y elaboran manualmente alimentos como las tortillas. Los hombres, por su parte, no todos tienen la suerte de trabajar en una fábrica dentro de la ciudad y deben viajar fuera para ocuparse en ranchos o en la producción agrícola.

Así, estamos frente a una población que tiene poco tiempo para dedicarlo a la "superación personal" o a los proyectos productivos como la elaboración de artesanías. Algo parecido fue la percepción de Felipe Ruiz mientras trabajó para la asociación:

En el Carlos Arroyo montan un invernadero tremendo, carísimo, con láminas de policarbonato, del que no se sacó nada. Hacen proyectos de chivas y gallinas, de cajeta, se consiguen una pasteurizadora [...] Y es que hacen proyectos de acuerdo a la necesidad que ellos ven, y no de acuerdo a la necesidad de otro (F. Ruiz, comunicación personal, I8 de abril, 20I7). 
En 20I6, la asociación redujo su personal a tres personas, cuando Ugalde -el director- pidió la renuncia a Estela y a Reyna. Quedaron entonces Arturo Ugalde, Eleazar Sánchez y Felipe Ruiz. Estela sigue a la fecha como voluntaria viviendo en el Díaz Infante y poco después, el presidente y el director decidieron vender las casas del Carlos Arroyo a sus residentes. Esto fue el punto de quiebre definitivo de Felipe Ruiz con la asociación:

Y yo me lavo las manos, ese no era el proyecto original. Mi idea del proyecto era servir a los indígenas no ir tras el dinero. Yo, antes de salir, firmé un documento en donde me aseguraba de que no se vendieran las casas del Díaz Infante (F. Ruiz, comunicación personal, I8 de abril, 20I7).

Este desacuerdo iba un poco más allá del lucro con las viviendas, Felipe Ruiz vio señales en las que podía haber un mal uso de los fondos en la Asociación.

\section{Asentamiento El Pájaro Azul}

Las vidas rarámuri se movían "como un río subterráneo”, horadando la piedra de las instituciones chabochi. Como el lector se habrá dado cuenta, el Pájaro Azul desapareció de esta narración tras la muerte de Díaz Infante y es que así fue también para la Compañía de Jesús y para la Asociación.

El Pájaro Azul se encuentra a unos tres kilómetros al oriente del Díaz Infante. Se llega a través de las mismas calles sin pavimentar -del otro lado de la carretera- en la colonia Sierra Azul, donde la mayoría de los inmuebles son granjas particulares. Sin embargo, hay más casas habitación o vecinos chabochi, a diferencia de los otros dos asentamientos cuyo aislamiento es notorio. Ya en el año 2016 -como en la actualidad- era notorio el contraste entre el Carlos Arroyo, que está completamente bardeado y acondicionado con un área común ordenada y limpia; mientras que el Pájaro Azul o Siyóname Churuwí 
cuenta con una precaria reja de alambre, la capilla luce abandonada con sus ventanas rotas y su campanario "vandalizado", con ilegibles caracteres "cholos"; en tanto que las casas están descuidadas y con sus puertas y sus ventanas maltrechas. Lo más llamativo son los jóvenes consumiendo solventes en la entrada del lugar.

Eleazar Sánchez, administrador de la Asociación, explica las diferencias entre el Pájaro Azul y los otros asentamientos de esta manera: "ese asentamiento se perdió, era un proyecto que estaba destinado a ser como el Carlos Arroyo, pero se perdió. Ahora hay asaltos, drogadicción, prostitución [...]" (E. Sánchez, comunicación personal, I3 de febrero, 20I7). Comentarios similares se repitieron después con Felipe Ruiz y con residentes del Carlos Arroyo y del Díaz Infante.

Desde mi perspectiva como autor, después de escuchar lo que se decía del asentamiento el Pájaro Azul, seguí visitándolo con cierta cautela, procurando acudir de día. Con el tiempo, mi impresión fue divergiendo de la que me habían transmitido. Identifiqué a los cuatro muchachos que consumen solventes en la entrada del lugar y se visten con atuendos típicos de las pandillas. Y ellos me identificaron a mí, de manera que dejamos de ser completos extraños. Hasta la fecha no he presenciado un hecho violento, ni prostitución. Estas impresiones las comparten los vecinos chabochi, con los que tuve oportunidad de charlar: el velador de una granja contigua, un hombre de unos 60 años, me dijo que nunca había tenido algún conflicto con ellos, pero sí señaló que "tienen muy fuerte el problema de drogas, pero es cosa de ellos, a nosotros no nos han hecho nada", también dijo que no conocía a nadie de los residentes de manera personal. La mujer de la tienda más cercana al asentamiento dijo que el asentamiento está "devastado por las drogas" y "sí trata uno de mantener sus distancias", pero también dijo que los rarámuri no robaban ni molestan a los vecinos. Sin duda, hay una divergencia entre mi percepción y las descripciones del Pájaro Azul ofrecidas por personas relacionadas con la Asociación, lo cual es importante dejar por sentada, so pena de parecer subjetivo. 
Tras la muerte de Carlos Díaz Infante, el lugar se volvió poco a poco incontrolable para las autoridades parroquiales. Como se mencionó antes, el asentamiento fue construido en un terreno ejidal y cuando se dio la oportunidad de las autoridades donara formalmente los tres terrenos que componían el asentamiento -en 20I0-, también se pensó que sería oportuno mejorar la infraestructura, ampliando las casas para equiparlas con baño y lavadero, pues los que tenía eran comunales.

La versión de Felipe Ruiz al respecto de los hechos que siguieron es que "se comete un gran error, se les dice que se van a mejorar las casa, pero que los borrachos, las prostitutas y los drogadictos, no van a volver... y ellos dijeron si sabes contar, no cuentes conmigo" (F. Ruiz, comunicación personal, I8 de abril, 20I7).

Cuando los problemas iniciaban en el asentamiento el Pájaro Azul, llegó de la Ciudad de México el hijo del siríame Camilo, quien tenía mucho tiempo en su cargo y había sido colocado ahí por Díaz Infante, pero su hijo había estudiado el seminario y de acuerdo a Felipe Ruiz, llegó "con aires de grandeza", asumió el papel de líder y dijo que los terrenos eran suyos porque se los había dado Carlos Díaz Infante. Instó a los residentes del Pájaro Azul para no salirse de sus casas. La gente se volvió hostil, hubo "dimes y diretes" y aunque los terrenos fueron regularizados y se pusieron a nombre de AsenTar A.C., la asociación decidió retirarse. También se fue la encargada del lugar, a la que Felipe Ruiz describe como una mujer "de dinero", "muy generosa, muy maternalista, muy proteccionista” (F. Ruiz, comunicación personal, i8 de abril, 20I7).

Después de que la asociación perdiera al Pájaro Azul, hubo un episodio en que trataron de recuperar documentos del padre Díaz Infante que se encontraban en la que había sido su casa. Ante la hostilidad de los residentes, pidieron el apoyo de la Coordinación Estatal de la Tarahumara para que mediara; sin embargo, no se les permitió entrar y el joven hijo Camilo, junto con "unos cholillos de pelos largos", los expulsó a pedradas. La funcionaria de la Coordinación "salió corriendo 
a su carro y a pelearse [...] porque venía tras ellos una horda de salvajes". Entonces se optó por dejar los documentos ahí, donde permanecen hasta la fecha (F. Ruiz, comunicación personal, i8 de abril, 20I7).

Doña Refugio, la actual siríame del Siyóname Churuwí, quien tiene 30 años viviendo en el lugar, ofrece una perspectiva diferente de lo ocurrido. Ella describió a la encargada del asentamiento como una mujer controladora que acaparaba de manera arbitraria las donaciones que recibían: "llegaban juguetes o dulces para los niños y ella los guardaba en un cuartito y ahí se quedaban". Estas afirmaciones respecto a los niños y la aversión que representa la encargada para Doña Refugio, resultan más interesantes si recordamos que los rarámuri suelen ser muy liberales con respecto al control que ejercen sobre los niños y niñas (towí y tewé). Les concede mucha autonomía y la manera en que se les educa es más bien una guía relativamente laxa en lugar de correctiva. Es difícil generalizar al respecto, pero hasta la fecha los testimonios y mi experiencia personal como investigador, me indican que esta es una tendencia: dejar hacer a los niños. Entonces, quitarles o retenerles juguetes o dulces podría resultar bastante irritante desde su perspectiva. Así pues, para Doña Refugio el conflicto con la asociación comenzó a partir de la presencia de la encargada del Siyóname Churuwí, por lo cual acudieron a la Coordinadora Estatal de la Tarahumara para pedirles que les siguieran "dando los apoyos, pero nomás, que aquí no los queremos [...] Ya ves que cuando se meten las asociaciones, pues nos traen el mal" (D. Refugio, comunicación personal, 20 de enero, 20I7).

La presencia del hijo de Camilo, este joven beligerante, también representó problemas al interior del asentamiento. Según los testimonios de Ruiz y de Doña Refugio, no solo se ocupó de formar alianzas con los jóvenes del Pájaro Azul, sino con cholos o pandilleros de fuera, chabochis; a quienes les permitió entrar, consumir drogas y operar desde el asentamiento. Esto causó mucho descontento y sensación de inseguridad, por lo que Doña Refugio acudió a la Coordinara -acompañada por otras mujeres del Pájaro Azul- para que 
les apoyara en la remoción de Camilo de su cargo como siríame. Así se hizo y Doña Refugio tomó el bastón de mando y expulsó al hijo de Camilo (D. Refugio, comunicación personal, 20 de enero, 20I7).

Con el tiempo, el Pájaro Azul alcanzó cierto nivel de paz y los cholos ya no operan en su interior. Sin embargo, la impresión de que se trata de un lugar muy peligroso y violento sigue viva entre los que presenciaron sus momentos críticos. Dijo Felipe Ruiz: "Ahí hay mucha borrachera, mucha drogadicción, prostitución [...] Y nadie hace por ellos nada [...] nosotros no pudimos entrarle como asociación [...]" (F. Ruiz, comunicación personal, I8 de abril, 20I7).

\section{El sector rarámuri}

La última parte de este relato se refiere al fraccionamiento Rinconada los Nogales y se trata de un espacio fundado por iniciativa rarámuri. Este núcleo residencial se encuentra en la misma parte de la ciudad que el Carlos Arroyo, el Díaz Infante y el Pájaro Azul, es decir, al suroeste de la ciudad de Chihuahua, en un polígono poco urbanizado, con carencias en infraestructura y servicios. Consta de 20 casas de construcción improvisada con madera y materiales reutilizados, donde viven alrededor de 60 personas. En este espacio se cumple al píe de la letra lo de "poco urbanizado" ya que no cuenta con instalaciones eléctricas y tienen una fuente provisional de agua potable mediante un tanque donado por la Junta Municipal de Agua y Saneamiento. Dos habitantes del lugar comentan que no cuentan con agua desde hace un año puesto que "se conectaron" varías fábricas y al parecer acaparan todo el suministro de la red.

136 En el asentamiento tiene lavaderos para la ropa, sanitarios y regaderas compartidas por todos los residentes. Las pequeñas edificaciones se encuentran repartidas de manera irregular en el que crecen lilas, duraznos y otros árboles. Por lo general se puede ver a los niños jugando a la pelota y a hombres y mujeres lavando ropa. Al norte de este pequeño núcleo residencial está el resto de la colonia Rinconada los 
Nogales, que cuenta con un centenar de casas de block, algunas de cartón y madera, y otras en obra negra. Aquí habitan chabochis de bajos recursos económicos, que -sin embargo- cuentan con luz eléctrica. A espaldas de este fraccionamiento se levantan los montones de desperdicios industriales que dejó la Fundidora de Ávalos en décadas atrás.

Este emplazamiento es resultado de un éxodo de resientes del Díaz Infante, que se produjo alrededor del año 2010 (M. Chacarito, comunicación personal, marzo 27, 20I7), tras el cual ocho familias se establecieron en Rinconada. Según comentarios de María Luisa, Felipe Ruiz les dijo: "Ya no pueden estar aquí, búsquense otro lugar donde vivir", lo cual puede sintetizar la impresión general de prepotencia con la que los rarámuri suelen ser tratados por la Asociación. Según Felipe Ruiz “en el Díaz Infante, siempre les sugerimos que buscaran otro lugar para crecer, pero cuando llegue ese momento es porque tienes algo igual o mejor, no pa'atrás", (F. Ruiz, comunicación personal, i8 de abril, 20I7).

El trámite de la propiedad de los terrenos comenzó en 2010 y continuaba en 20I7. Así pues, no es un lugar establecido por ninguna asociación civil o alguna dependencia gubernamental, aunque su historia se encuentra vinculada a la del resto de los asentamientos de esta área, fundados por iniciativa de los jesuitas.

María Luisa Chacarito, a lado de su hermana Anís Isabel, fueron de las primeras residentes y cuentan que para poder asentarse tuvieron que acudir a "hacer presencia", esperando muchas horas, durante varios días, bajo el sol de un verano especialmente caluroso, para que cuando fuera gente del gobierno ellos estuvieran ahí y pudieran pedir los terrenos. Los instruyó para hacer esto un tal Pepe Borunda, quien ha sido el intermediario entre los residentes chabochi de Rinconada y el gobierno municipal. Se trata de un líder de "paracaidistas", es decir, participa en la regularización de terrenos cuyos habitantes no cuentan 
con título de propiedad (M. Chacarito, comunicación personal, marzo 27, 2017).

En algún momento María Luisa decidió prescindir de la mediación del señor Borunda y optó por gestionar ella misma, junto con su hermana, lo referente a los títulos de propiedad. Actualmente ellas y otros residentes acuden a frecuentes juntas, con distintas dependencias del gobierno estatal y municipal, esperando les vendan o les donen los terrenos, se hagan las instalaciones eléctricas necesarias y también que se construya un muro perimetral que les brinde seguridad, ya que ha ocurrido que pandilleros o drogadictos irrumpen en las casas. Aquí vemos como los rarámuri por su propia iniciativa buscan la consolidación de un espacio exclusivo y cerrado para su residencia, acaso una innovación en sus tendencias colectivas, fruto de la estancia y el contacto con aquellos emplazamientos cerrados como El Oasis, el Díaz Infante y el Carlos Arroyo (F. Ruiz, comunicación personal, i8 de abril, 20I7).

Los residentes de este asentamiento acordaron que no formarán un grupo de autoridades (siríame, segundo siríame, capitán, teniente) como sí se hace en los asentamientos antes mencionados. Por un lado, esta es una manera de diferenciarse, ya que a los rarámuri de Rinconada no quieren que su espacio sea etiquetado con el mote de "asentamiento", aunque ellos mismos lo llamen así de vez en cuando. Su objetivo es salirse de los esquemas de control y vigilancia propuestos por la Asociación y expresamente eligieron marcar una línea entre ellos y los chabochi de Rinconada, nombrándose "sector rarámuri", término que los diferencia, pero que no está asociado a una condición excepcional, que alude a una población migrante que se asienta en algún territorio diferente al propio.

Otro aspecto que se puede leer, a partir de la negativa de formar un grupo de autoridades, lo señala María Luisa Chacarito, quien fue parte del cuerpo de autoridades rarámuri con los que trabajaba la asociación, cuando afirma que es innecesario que el Sector Rarámuri de Rinconada 
elija un siríame, porque su impresión es que este tipo de autoridades le son más útiles al estado chabochi que a los rarámuri. Para la Asociación y para las dependencias gubernamentales -como lo fue en el periodo colonial para la Iglesia y el Imperio Español (Deeds, 2003)- las autoridades rarámuri han tenido un papel indispensable como mediadores entre ellos y los poderes exógenos. En la actualidad la Comisión para los Pueblos Indígenas exige a los asentamientos establecer un grupo "oficial" y fijo de tres autoridades, que tienen que registrar ante ellos. Los rarámuri de Rinconadas se opusieron abiertamente a esta medida y han sido amenazados con dejar de recibir los apoyos gubernamentales, que son tan necesarios en la vida cotidiana de las familias cuya residencia en la ciudad está en proceso de consolidación.

\section{Comentarios finales}

Como se puede observar, una constante a lo largo del texto es la intervención institucional que ocurre cotidianamente en los espacios de vida de los rarámuri urbanos. A su vez, un aspecto importante de esta injerencia es la promoción de diferentes proyectos educativos en los asentamientos indígenas, algunos de los cuales son mencionados en la anterior narración. Sin embargo, estas iniciativas palidecen en comparación con el impacto que tiene la constitución misma de estos espacios en la formación de la subjetividad de los rarámuri urbanos.

Los asentamientos, como resultado de la interacción entre las instituciones no-indígenas y los rarámuri que migran y residen en la ciudad, constituyen en sí mismos espacios de educación formal e informal, así como lugares en los que se configuran las nuevas subjetividades rarámuri.

El reconocimiento de que los residentes de los asentamientos pueden ser rarámuri y al mismo tiempo habitantes establecidos en la ciudad, implica para la población no-indígena el rompimiento de etiquetas y prejuicios que los encasillan en la vida rural. Así, se espera que el 
presente texto, además de ilustrar las condiciones en que surgieron y se desarrollaron los asentamientos, sirva como un referente para conocer la manera en que la subjetividad de los rarámuri urbanos se consolidó desde finales del siglo XX.

\section{Referencias}

Asentamientos Tarumares. [Alfonso López Collada]. (20I5, 3 de diciembre). Asentamientos Tarahumares AC, Chihuahua [Archivo de Video]. Recuperado de: https://www.youtube.com/watch?v=DPnUCYb5piY\&t=Is

Chacarito, M.L. (2017, I3 de marzo). Entrevista realizada por Nelson Solorio Talavera. Chihuahua, México.

Cramaussel, C. (I99I). Encomiendas, repartimiento y conquista en Nueva Vizcaya. Historias, (25), 73-86.

Deeds, S. (2003). Defiance and deference in Mexico's colonial North. Estados Unidos: University of Texas.

Fernández Ramos, M. de G. (2013). La participación de las mujeres rarámuri en el proceso de inserción residencial y laboral en la ciudad de Chihuahua. Mexicali, México: Instituto de Investigaciones Culturales.

Herrera Bautista, Arturo (2013). La vida en los intersticios. Estrategias identitarias de los rarámuri en la ciudad de Chihuahua. Baja California, México: Instituto de Investigaciones Culturales.

Lara Meza, A. M. (20I0). La construcción de la memoria como fuente. En A.M. Lara Meza, F. Macías Gloria y M. Camarena Ocampo (Coords.), Los oficios del historiador. Taller y prácticas de la historia oral (pp. 59-78). Guanajuato México: Universidad de Guanajuato. 
Mireles, E. (2012). La Compañía de Jesús en la Tarahumara. Recuperado de: https://jesuitasentarahumara.wordpress.com/2012/07/03/lacompania-de-jesus-en-la-tarahumara/

Morales Muñoz, M.V. (2013). Las prácticas de intervención institucional en la creación y organización sociopolítica de los asentamientos rarámuris en la ciudad de Chihuahua. El caso de El Oasis. Relaciones, 34(I34), I9-55.

Morales Muñoz, M.V. (20I4). "Aquí la mujer se siente más responsable”. Género y etnicidad rarámuri en la Ciudad de Chihuahua. Entre las relaciones de complementariedad $y$ de desigualdad [Tesis doctoral]. Centro de Investigaciones y de Estudios Superiores en Antropología Social, México. Recuperado de: http://repositorio.ciesas.edu.mx/bitstream/handle/I23456789/25 2/D248.pdf?sequence $=$ I\&isAllowed $=y$

Moss, W. M. (I99I). La historia oral: ¿qué es y de donde proviene? En D. Schwarsztein (comp.), La Historia oral (pp. 2I-35). Buenos Aires, Argentina: Centro Editor de América Latina.

Plascencia, J.C. (2017, 20 de marzo). Entrevista realizada por Nelson Solorio Talavera. Chihuahua, México.

Refugio, D. (20I7, 20 de enero). Entrevista realizada por Nelson Solorio Talavera. Chihuahua, México.

Ruiz, F. (2017, I8 de abril). Entrevista realizada por Nelson Solorio Talavera. Chihuahua, México.

Sánchez, E. (20I7, I3 de febrero). Entrevista realizada por Nelson Solorio Talavera. Chihuahua, México 
Servín Herrera, E. (2002). Ralámuli Ra’ichábo! ¡Hablemos el tarahumar! [Colección Solar]. Chihuahua, México: Instituto Chihuahuense de la Cultura.

Ugalde, A. (20I4, 24 de enero). Equidad en la difusión de OSC, entrevistador Jesús Arizmendi Valdez [Programa de radio]. México.

\section{Notas}

I La palabra chabochi significa en el idioma de los rarámuri "no-indígena", ya sea mestizo o europeo. Etimológicamente, alude a la barba, el vello facial. Mientras que la palabra "rarámuri" (también escrita "ralámuri"), significa "persona" o "gente", en el habla coloquial de los rarámuri. Para más información sobre los significados y la pronunciación adecuada de estos vocablos se puede consultar Ralámuli Ra'ichábo! ¡Hablemos Tarahumar! (Servín, 2002).

${ }^{2}$ Felipe Ruíz es un laico miembro de la Compañía de Jesús, quien operó como director del asentamiento Díaz Infante a partir del 2006 y hasta el 2016.

${ }^{3}$ Carlos Arroyo Herrera quedó a cargo de la Parroquia de San José de los Tarahumaras.

${ }^{4}$ María Luisa Chacarito es una rarámuri que participó de manera directa en la negociación de las reglas bajo las cuales habrían de vivir los rarámuri al interior del Díaz Infante.

${ }^{5}$ Siríame es la autoridad rarámuri, hombre o mujer, elegida por el consenso de los residentes de una localidad rarámuri. Por lo general cumple el papel de mediar entre la colectividad rarámuri y las instituciones no-indígenas.

${ }^{6}$ AsenTar A.C. contaba con secretarias y asistentes, así como oficinas en el centro de la ciudad de Chihuahua.

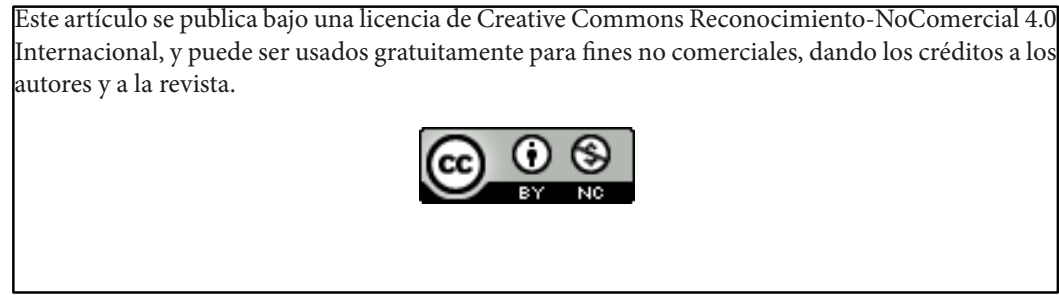

